\title{
Lining Up for Wakefield Acceleration
}

\section{A proposed design for plasma accelerators would use line-focused laser pulses to overcome the problem of particles outrunning the acceleration region.}

\section{by Jeroen van Tilborg ${ }^{1}$}

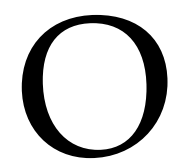
ne way to accelerate particles is to fire an intense laser pulse into a plasma, creating a density wake whose electric field pushes charged particles like electrons to high speeds [1]. The accelerating gradient from such a plasma wakefield is much higher than can be achieved in conventional radio-frequency-based technology. However, a central difficulty with employing laser wakefield accelerators (LWFAs) is that the electrons eventually outrun the accelerating region of the laser-driven wakefield. Because of this dephasing, a single stage of wakefield acceleration is typically limited to a few tens of centimeters in length, and it is forced to operate at a low density, which constrains the accelerating gradient. Several ideas have been proposed to deal with this dephasing limit. A new idea from John Palastro and colleagues from

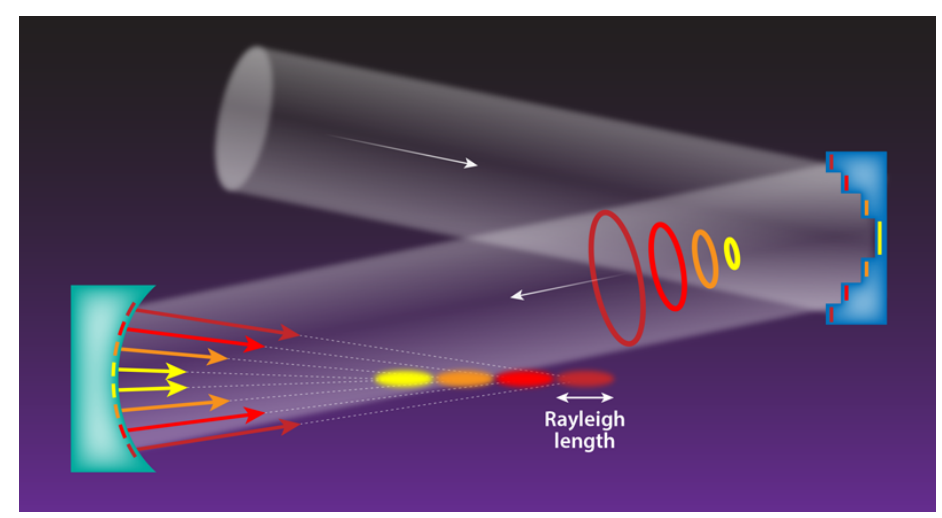

Figure 1: The proposed dephasingless wakefield acceleration would utilize two optical elements: an echelon (blue) and an axiparabola (green). A laser pulse would first strike the echelon, producing a series of time-staggered rings. The axiparabola would then focus these rings onto a line, consisting of multiple

Rayleigh-length segments. This spatiotemporal laser control could generate a wakefield that travels at the speed of light, overcoming one of the main limitations to wakefield acceleration. (APS/Alan Stonebraker)

${ }^{1}$ BELLA Center, Lawrence Berkeley National Laboratory, Berkeley, CA, USA the University of Rochester, New York, uses special optical components to focus the laser into points along a line, thus extending the wake region so electrons are accelerated for longer and at a higher gradient [2]. This method faces some hurdles, such as increasing the laser power above what's currently available, but it has the potential to accelerate electrons to $\mathrm{TeV}$ energies over just a few meters.

The high gradients offered by laser wakefield acceleration open the path for tabletop accelerators at universities, hospitals, and smaller R\&D labs, with applications such as $x$-ray bio-imaging, active nuclear interrogation, and medical treatments. LWFA also enables progress towards future $\mathrm{TeV}$-scale particle colliders that promise to extend our understanding of the basic structure of the Universe [3]. In the past 20 years, the LWFA community has evolved from smallscale proof-of-principle demonstrations to milestones in the production of beams with high current, low emittance, and narrow (percent-level) energy spread. Precision control of the laser-plasma interaction with multiple lasers or density profile shaping is a central thrust in ongoing research. Efforts are also underway to increase the number of laser shots from a few per second to more than a thousand per second, which will benefit stabilizing feedback procedures and applications that require high flux.

The maximum achievable electron energy for LWFA is determined by the accelerating field strength and the length of acceleration $L_{a c c}$, both of which are limited by laser and plasma physics [1]. The field strength scales with the plasma density as $E_{z} \sim n^{1 / 2}$. At currently used densities $\left(10^{16}-10^{19} \mathrm{~cm}^{-3}\right)$, the field strength can exceed $10-100$ $\mathrm{GV} / \mathrm{m}$, which is about a thousand times the acceleration in traditional radio frequency cavities used at places like CERN. $E_{z}$ also depends on the laser, whose intensity is determined by the laser energy, wavelength, pulse duration, and spot size.

The effective accelerator length depends on the laser as well. Specifically, the acceleration is limited to the region over which the laser remains focused. In vacuum, this focus region is characterized by the Rayleigh length $z_{R}$, which is calculated with the laser wavelength and spot size. For most setups, the Rayleigh length is in the millimeter to centimeter range, but researchers can extend the focusing region by adding light-guiding structures, as was demonstrated in a recent LWFA experiment that achieved 8-GeV acceleration 
energies with a 20-cm-long waveguide [4]. However, guiding structures are not without challenges, including the need for precise alignment and transverse mode control to avoid damage from the high-power laser pulses.

Guiding structures also don't alleviate the problem of dephasing. Dephasing occurs because the wakefield region travels behind the laser at a speed slower than the vacuum speed of light, so electrons accelerated to relativistic energies will eventually get ahead of it. As such, the wakefield speed places an upper limit on the acceleration length, which scales with density as $n^{-3 / 2}$. To increase this limit - and correspondingly increase the maximum electron energy - traditional setups use a low plasma density.

Recently, a group proposed a scheme to overcome this dephasing limit by obliquely intersecting two tilted-pulse-front lasers [5]. The interference between the lasers would generate a wakefield region that travels at the speed of light in vacuum. However, this approach requires precision control of two lasers, with potential challenges from transverse wakefield asymmetries.

Palastro et al. [2] have a similar idea for speeding up the wakefield region that uses a single laser and special optics rather than interfering lasers. Specifically, their proposed method combines an echelon (a mirror with specially designed steps) and an axiparabola (a recently developed curved mirror) [6]. As the team conceives it, a laser pulse would first strike the echelon, which divides the light into a number of concentric rings (Fig. 1). These rings would be separated in time, such that the outer rings arrive at the axiparabola ahead of the inner rings. The curved reflective surface of the axiparabola would focus the rings at successive points along a line. This spatiotemporal shaping of the laser pulse offers a way to generate a wakefield traveling at the vacuum speed of light, which would circumvent dephasing.

To see how this "dephasingless" LWFA compares to previous schemes, we can imagine the system is tuned so that each ring produces the same acceleration effect as a single laser pulse in the traditional nonguided LWFA. In other words, each ring generates a focal segment that is one Rayleigh-length long, and if there are $N$ rings, the total acceleration length would be $N$ times the Rayleigh length. In addition to increasing the acceleration length, dephasingless LWFA could operate at high plasma density, which means a higher accelerating gradient. The bottom line is that dephasingless LWFA could potentially reach higher electron energies than previous methods with the same acceleration length.

One potential hurdle to this proposal is the laser energy requirements. If each of the $N$ rings is to produce a separate wakefield, then the single laser pulse would need roughly $N$ times more energy than in a traditional LWFA of compa- rable laser intensity. For TeV-scale electron acceleration, the number of rings would need to be in the thousands, and the corresponding femtosecond-duration laser energy would be multiple kilojoules, which may pose a challenge in terms of the availability of laser systems and power consumption limitations (especially for $\mathrm{kHz}$ operation). There are other issues that may require further investigation, such as how well does the line-focus scale to large ring numbers, and how will the spatiotemporal laser evolution affect wakefield generation and laser-electron overlap. In addition, questions remain over the inverse accelerated charge scaling with plasma density [7].

Despite these uncertainties, Palastro et al. have provided a clever variation of staged acceleration, with independently timed laser "beamlets" driving acceleration without the need for intrastage optics or complex guiding structures. As such, dephasingless LWFA enables new opportunities in the choice of density and laser-plasma accelerator architecture. This could be of particular importance for leveraging future multipetawatt lasers to compactly create high-energy electron beams that can probe nonlinear quantum electrodynamics [8]. Next on the dephasingless LWFA to-do list: performing detailed simulations of laser delivery and wakefield production at multi-GeV scale and testing the waters regarding practical limitations.

This research is published in Physical Review Letters.

\section{REFERENCES}

[1] E. Esarey et al., "Physics of laser-driven plasma-based electron accelerators," Rev. Mod. Phys. 81, 1229 (2009).

[2] J. P. Palastro et al., "Dephasingless laser wakefield acceleration," Phys. Rev. Lett. 124, 134802 (2020).

[3] W. P. Leemans and E. Esarey, "Laser-driven plasma-wave electron accelerators," Phys. Today 62, No. 3, 44 (2009).

[4] A. J. Gonsalves et al., "Petawatt laser guiding and electron beam acceleration to $8 \mathrm{GeV}$ in a laser-heated capillary discharge waveguide," Phys. Rev. Lett. 122, 084801 (2019).

[5] A. Debus et al., "Circumventing the dephasing and depletion limits of laser-wakefield acceleration," Phys. Rev. X 9, 031044 (2019).

[6] S. Smartsev et al., "Axiparabola: A long-focal-depth, highresolution mirror for broadband high-intensity lasers," Opt. Lett. 44, 3414 (2019).

[7] W. Lu et al., "Generating multi-GeV electron bunches using single stage laser wakefield acceleration in a 3D nonlinear regime," Phys. Rev. STAB 10, 061301 (2007).

[8] A. Di Piazza et al., "Extremely high-intensity laser interactions with fundamental quantum systems," Rev. Mod. Phys. 84, 1177 (2012).

10.1103/Physics.13.49 